\title{
Fraunhofer to mine Sanofi microbial collection
}

Paris-based Sanofi and the Fraunhofer Institute for Molecular Biology and Applied Ecology (IME) in January announced a joint effort to explore natural products as leads for developing new therapeutics, particularly drugs for treating infectious diseases. Many observers welcome this renewed interest in the natural products, saying there is plenty of untapped value to be uncovered but also warning that this route promises no short cuts.

The alliance with the Aachen, Germanbased Fraunhofer is Sanofi's second foray into natural products. The first was in 2011, when the big pharma forged a similar but broader deal with Warp Drive Bio. The Cambridge, Massachusetts biotech secured \$125 million upfront, and equity investment of up to $\$ 75$ million from a consortium comprising Sanofi, Boston-based venture capital firm Third Rock Ventures and Greylock Partners of Menlo Park, California (Nat. Biotechnol. 30, 385-387, 2012). That agreement launched that company on a mission to comb through genomic signatures of microbes to uncover hidden natural products.

The Sanofi and Fraunhofer research team, headed by Peter Hammann of Sanofi and Andreas Vilcinskas from Fraunhofer, will focus entirely on Sanofi's collection of more than 100,000 different microorganisms. A group of at least 16 scientists will be analyzing these microbial strains, cultivating them under various conditions, stimulating them to produce active substances and investigating their effects. Separately, several other companies are delving into natural products as a source for antibiotics or drugs for treating other medical conditions, including Cubist near Boston, Eisai of Tokyo and Woodcliff Lake, New Jersey, PharmaMar near Madrid, and Novartis in Basel.

For drug developers, revisiting natural products is like a widower swooning once again over a high school sweetheart. The earliest antimicrobials were uncovered from soil-dwelling microorganisms. But interest in natural products dwindled and pharma companies eventually dropped that approach in favor of medicinal chemistry. Later, in the heated quest for new drug candidates, approaches became ever more dazzling - combinatorial chemical libraries, genomics and high-throughput screening. But none of these strategies proved as fruitful as the early explorations.

"Access to Sanofi's natural product collection will also create value for other industries," says Rainer Fischer, senior executive director from Fraunhofer IME. For antibiotics expert Karen Bush, formerly with Johnson \& Johnson and now at Indiana University in Bloomington, the project is worth watching. "The resources available in the Sanofi-Fraunhofer alliance will allow experienced investigators to mine a valuable set of organisms for identification of novel compounds that may serve as the basis for semi-synthetic drug optimization." Natural products, she adds, "were abandoned by large pharma in the 1990s" when they deemed the analysis of prospective compounds for signs of activity "too costly and too time consuming."

The Sanofi-Fraunhofer alliance "is a welcome and timely initiative," says Alan Harvey of the University of Strathclyde in Glasgow, who is part of the team coordinating SeaBioTech, a natural products $R \& D$ consortium that includes several biotech companies across Europe. "Natural products continue to be valuable as sources of leads to new medicines even though big pharma has almost completely withdrawn from the area. In terms of anti-infective drugs, about $70 \%$ of antibiotics are natural products or semisynthetic derivatives."

Whether the Sanofi-Fraunhofer partnership succeeds, "depends on how they search through the strain collection," says New Jersey-based antibiotic development consultant Lynn Silver. "Without quite a bit of

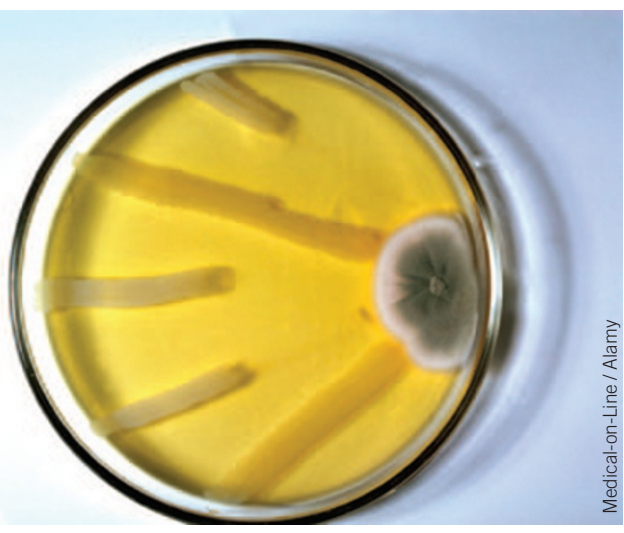

A natural product makes history. A penicillin culture from the $A$ Flemming collection.

hands-on experience in anti-bacterials, they will go in circles and reinvent wheels for a long time before getting anywhere. A number of companies are doing various kinds of bioprospecting, and no one has much of anything-no major breakthroughs. Unless you do creative screening, it is very hard to find anything novel and safe."

Yet, drug developers broadly speaking "need an infusion of new chemical diversity," says William Fenical of Scripps Institution of Oceanography at the University of California, San Diego, who leads a natural products research group focused on marine organisms. "It's encouraging that Sanofi is now seeking to return to the field of natural microbial products, which for 50 years was extraordinarily productive."

\section{Jeffrey L Fox Washington, $D C$}

\section{My kingdom for a DNA sequencer}

King Richard III will be the first royal to be sequenced in a project spearheaded by the Wellcome Trust and the Leverhulme Trust. The 500-year old bones were found without a coffin under a parking lot in Leicester during an archaeological dig in September 2012. The DNA sequencing effort, directed by Turi King, the geneticist who identified the Plantagenet king, will take at least a year and cost over $\$ 160,000$. The results might reveal whether the scoliosis that contorted his back was genetic, and his susceptibility to disease. But whether the monarch sent his young nephews, the little princes in the Tower, to their deaths will remain unresolved.

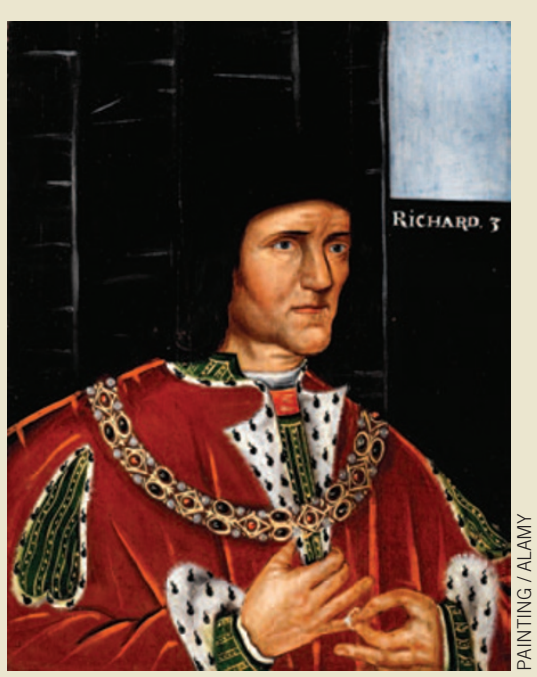

